\title{
Ectopic mineralization of cartilage and collagen-rich tendons and ligaments in Enpp1asj-2J mice
}

\author{
Jieyu Zhang ${ }^{1,2}$, Nathaniel A. Dyment ${ }^{3}$, David W. Rowe ${ }^{3}$, Sarah Y. Siu' ${ }^{1}$ John P. \\ Sundberg ${ }^{4}$, Jouni Uitto ${ }^{1}$ and Qiaoli $\mathbf{L i}^{1}$ \\ ${ }^{1}$ Department of Dermatology and Cutaneous Biology, The Sidney Kimmel Medical College at Thomas Jefferson University, \\ Philadelphia, PA, USA \\ ${ }^{2}$ Department of Dermatology, The Fourth Military Medical University, Xijing Hospital, Xi'an, China \\ ${ }^{3}$ Center for Regenerative Medicine and Skeletal Development, University of Connecticut Health Center, Farmington, CT, USA \\ ${ }^{4}$ The Jackson Laboratory, Bar Harbor, ME, USA \\ Correspondence to: Qiaoli Li, email: Qiaoli.Li@jefferson.edu
}

Keywords: ectopic mineralization, tendon and ligament calcification, chondrocalcinosis, generalized arterial calcification of infancy, mouse models, Pathology Section

Received: October 22, $2015 \quad$ Accepted: January 31, 2016

Published: February 17, 2016

\section{ABSTRACT}

Generalized arterial calcification of infancy (GACI), an autosomal recessive disorder caused by mutations in the ENPP1 gene, manifests with extensive mineralization of the cardiovascular system. A spontaneous asj-2J mutant mouse has been characterized as a model for GACI. Previous studies focused on phenotypic characterization of skin and vascular tissues. This study further examined the ectopic mineralization phenotype of cartilage, collagen-rich tendons and ligaments in this mouse model. The mice were placed on either control diet or the "acceleration diet" for up to 12 weeks of age. Soft connective tissues, such as ear (elastic cartilage) and trachea (hyaline cartilage), were processed for standard histology. Assessment of ectopic mineralization in articular cartilage and fibrocartilage as well as tendons and ligaments which are attached to long bones were performed using a novel cryohistological method without decalcification. These analyses demonstrated ectopic mineralization in cartilages as well as tendons and ligaments in the homozygous asj$2 \mathrm{~J}$ mice at 12 weeks of age, with the presence of immature osteophytes displaying alkaline phosphatase and tartrate-resistant acid phosphatase activities as early as at 6 weeks of age. Alkaline phosphatase activity was significantly increased in asj-2J mouse serum as compared to wild type mice, indicating increased bone formation rate in these mice. Together, these data highlight the key role of ENPP1 in regulating calcification of both soft and skeletal tissues.

\section{INTRODUCTION}

Ectopic mineralization, characterized by deposition of calcium hydroxyapatite complexes on soft connective tissues, is a common age-associated phenomenon. Depending on the inflicted tissue, these acquired pathological processes can cause considerable morbidity [1]. Ectopic mineralization of cartilage and collagen-rich tendons and ligaments can be a cause of considerable orthopedic problems resulting in limitations in mobility due to tissue degeneration, and often requiring medical or surgical intervention [2,3]. Mechanistically, two major types of ectopic mineralization processes have been recognized [4]. First, metastatic calcification refers to deposition of calcium complexes as a result of elevated serum levels of calcium and/or phosphate, while dystrophic calcification is secondary to some form of insult to the tissues, frequently observed as a result of trauma and inflammatory diseases. In dystrophic calcification, serum calcium/phosphate levels are normal. Thus, ectopic mineralization can represent the consequences of several contributing metabolic and environmental factors, making the uncovering of precise basis of these disorders exceedingly difficult.

Several Mendelian heritable disorders depict phenotypic similarities with acquired forms of metastatic 
or dystrophic calcification, and many of these conditions serve as genetically controlled models to study various facets of pathological mineralization [5]. Such conditions include pseudoxanthoma elasticum, which demonstrates mineralization in the skin, eyes and the cardiovascular system, as a result of mutations in the ABCC6 gene [6]. Another very severe ectopic calcification disorder affecting arterial blood vessels and depicting joint and spine ossification is generalized arterial calcification of infancy (GACI), harboring mutations in the ENPP1 gene $[7,8]$.

Mouse models corresponding to several heritable ectopic mineralization disorders have been extremely helpful in identifying critical pathways involved. In addition, these model systems have provided evidence of intricate pro-mineralization/anti-mineralization networks in peripheral connective tissues necessary to maintain normal homeostasis preventing ectopic mineralization [5]. While the focus on characterization of these mice has been on mineralization affecting the skin and the cardiovascular system, less attention has been made on ectopic mineralization of cartilage and periarticular tendons and ligaments, major issues encountered in various orthopedic disorders.

One of the recently characterized mice mimicking GACI is the Enpp $1^{a s j-2 J}$ mouse (referred to hereon as asj$2 J$ mouse). This mouse arose spontaneously in a largescale production colony of BALB/cJ mice at The Jackson Laboratory [9]. These mutant mice develop abnormal forepaw position and gait due to stiffening of the joints, a phenotype similar to a previously characterized asj ("ages with stiffened joints") mouse harboring p.V246D mutation in the Enppl gene [10]. Therefore, this mutant mouse was named as asj-2J being allelic to asj mouse.
The asj-2J mice carry a large, $40,035 \mathrm{bp}$, deletion from intron 1 to 3' UTR of the Enppl gene, coupled with a 74 bp insertion [9]. Plasma PPi concentration and $\mathrm{PPi} / \mathrm{Pi}$ ratio was significantly reduced in homozygous asj-2J mice. As a consequence of this spontaneous mutation, extensive mineralization of the arterial vasculature and the dermal sheath of vibrissae was demonstrated by a combination of histopathology with calcium-specific stains, direct chemical assay of calcium, and microcomputed tomography. We have now further characterized the asj-2J mouse with particular focus on mineralization of cartilage and collagen-rich ligaments and tendons, with clinical relevance to orthopedic conditions.

\section{RESULTS}

\section{Experimental design}

Previous studies demonstrated that feeding asj-2 $\mathrm{J}$ mice with "acceleration diet", enriched in phosphate $(2 \mathrm{x})$ and reduced in magnesium $(20 \%)$ content in comparison to control diet, results in acceleration of the ectopic mineralization [9]. We, therefore, placed Enpp1 $1^{+/+}$, Enpp $1^{+/ a s j-2 J}$ and Enpp $1^{a s j-2 J}$ mice on either a standard control rodent diet or acceleration diet. Two experimental designs were utilized in different groups of mice (Table 1 ). In the first set of experiments (Set 1), 12-week-old asj$2 J$ mice were examined for ectopic mineralization and subjected to blood analysis, in comparison to wild type and heterozygote littermates of the same age. To determine the onset of mineralization on these two different diets, a second set of homozygous asj-2J mice was examined at
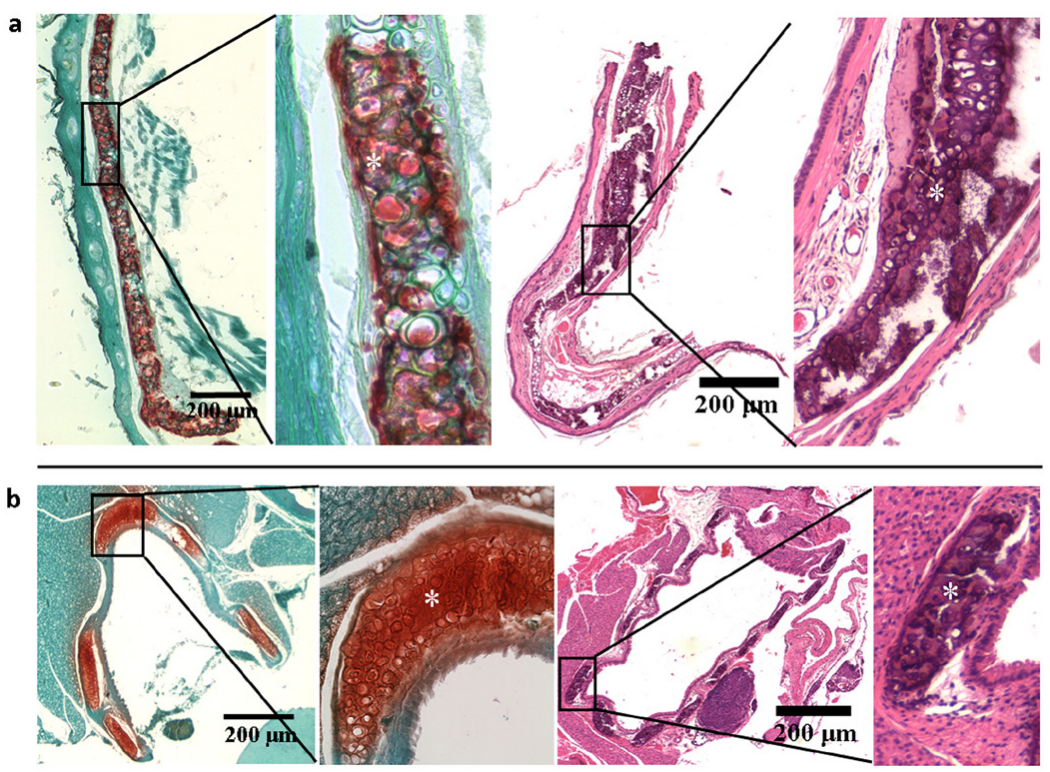

Figure 1: Extensive mineralization of elastic and hyaline cartilages in the asj-2 $J$ mice at 12 weeks of age (white asterisk). Alizarin red staining (left panel) and H\&E staining (right panel) reveal extensive mineralization in the elastic cartilage of outer ear (a) and hyaline cartilage of trachea (b) of asj- $2 J$ mice. 
different earlier time points (Set 2).

\section{Histopathologic evaluation of mineralization}

Three types of cartilage, hyaline, elastic, and fibrocartilage, with different connective tissue composition and organ distribution were analyzed histologically [11]. Histopathological examination of the outer ear and trachea in formalin fixed paraffin sections with Alizarin red and H\&E staining procedures revealed extensive calcification of elastic cartilage and hyaline cartilage, respectively, in $a s j-2 J$ mice at 12 weeks of age when kept on normal or acceleration diet (Figure 1).

Assessment of mineralization in articular cartilage and fibrocartilage as well as tendons and ligaments which are attached to long bones was technically difficult using regular fixation and sectioning procedures due to inability to section mineralized tissues. Therefore, we used a cryohistological method without decalcification of bones $[12,13]$. This method is based on the use of cryotape that adheres to the tissue section, which then allows for sectioning of calcified tissues while maintaining tissue morphology. Coronal sections of spine, knee, foot and shoulder were obtained with this methodology. Toluidine Blue $\mathrm{O}$ or Alizarin red staining showed aberrant cartilage, tendon and ligament mineralization in intervertebral discs, patellar tendons, meniscii, and supraspinatus tendons of 12-week-old $a s j-2 J$ mice (Figure 2). Toluidine Blue $\mathrm{O}$ and Alizarin red staining of sagittal sections of the knee and ankle showed posterior/anterior mineralization of cruciate ligaments, meniscus, lateral/medial collateral ligaments and Achilles tendon in 12-week-old $a s j-2 J$ mice (Figure 2). a

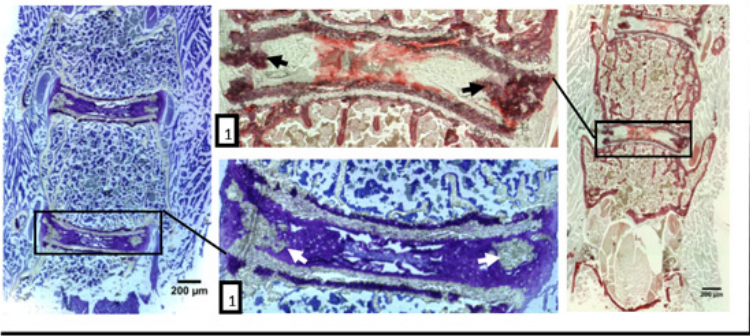

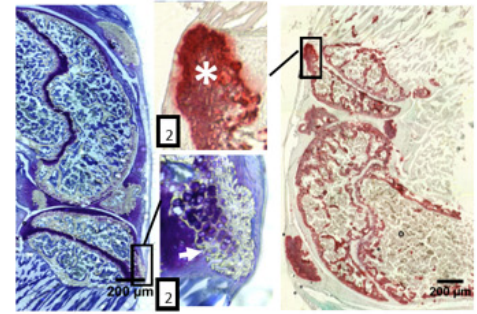

b

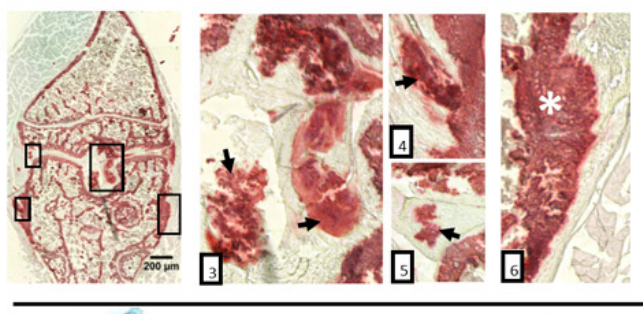

c
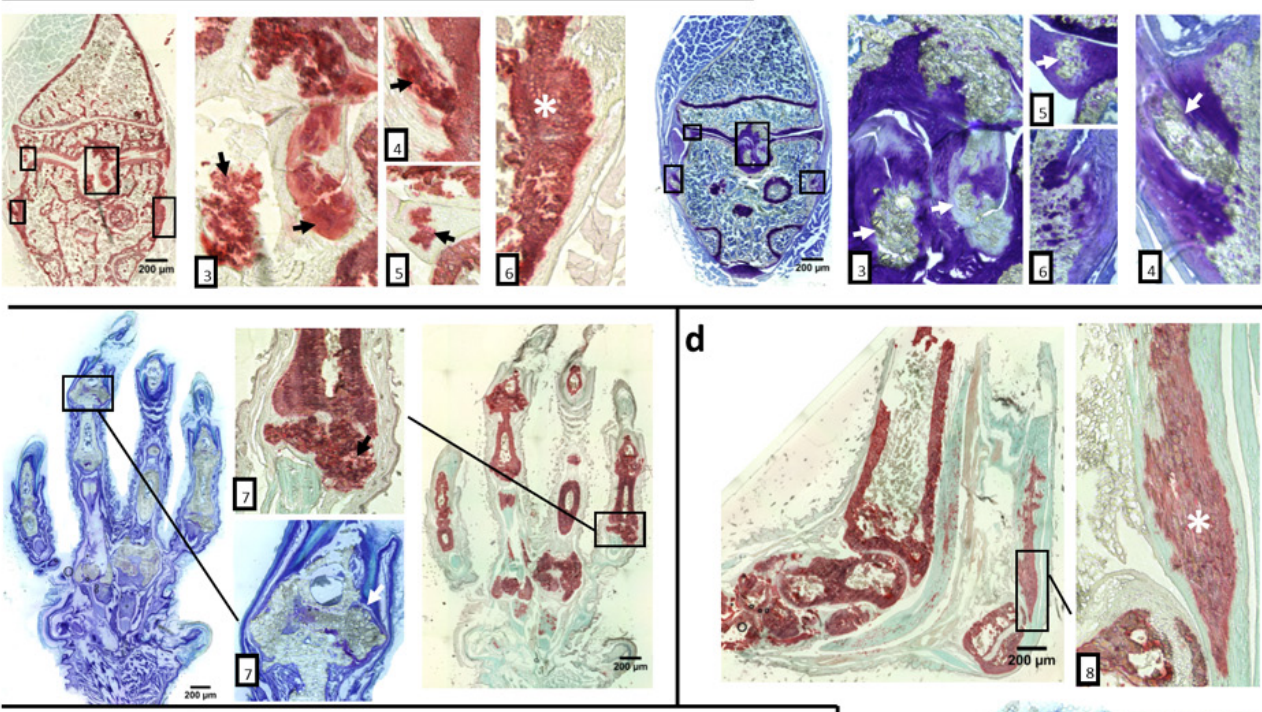

d
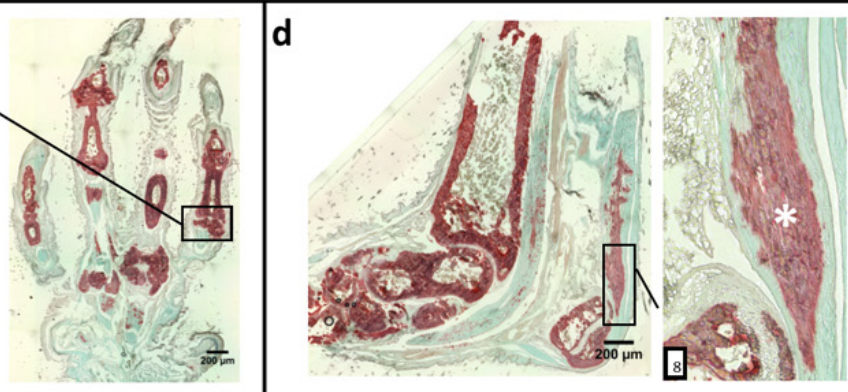

e
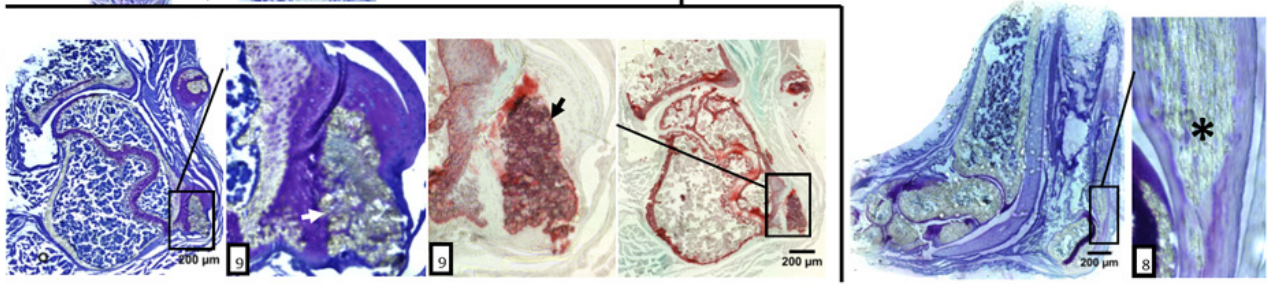

Figure 2: Aberrant cartilage and ligament mineralization in asj-2 $J$ mice at 12 weeks of age. Toluidine Blue $O$ staining (blue \& purple) and Alizarin red staining (red \& green) reveal extensive cartilage and ligament mineralization in the intervertebral disc, knee, foot, ankle and shoulder of $a s j-2 J$ mice, marked by arrow or asterisk. a. Coronal sections of spine show mineralization in intervertebral discs and posterior longitudinal ligament (1). b. Coronal and sagittal sections of the knee show mineralized in patellar tendons (2), posterior/anterior cruciate ligaments (3), meniscus (5) and lateral/medial collateral ligaments $(4,6)$. c. Coronal sections of the foot show the hypertrophied mineralized joints (7). d. Sagittal plane of the ankle shows the Achilles tendon mineralization (8). e. Coronal sections of shoulder show supraspinatus tendon mineralization (9). 
Table 1: Experimental design for analysis of $a s j-2 J$ mice

\begin{tabular}{|c|c|c|c|}
\hline Group & Genotype & Age (weeks) & Number of mice examined (female, male) \\
\hline \multicolumn{4}{|l|}{ Set 1: } \\
\hline Normal diet & Enpp1 $1^{+/+}$ & 12 & 4,3 \\
\hline Normal diet & Enpp $1^{+/ a s j-2 J}$ & 12 & 3,5 \\
\hline Normal diet & Enpp $1^{a s j-2 J}$ & 12 & 2,3 \\
\hline Acceleration diet & Enpp1 $1^{+/+}$ & 12 & 5,4 \\
\hline Acceleration diet & Enpp $1^{+/ a s j-2 J}$ & 12 & 3,3 \\
\hline Acceleration diet & Enpp $1^{\text {asj-2J }}$ & 12 & 4,2 \\
\hline \multicolumn{4}{|l|}{ Set 2: } \\
\hline Normal diet & Enpp $1^{a s j-2 J}$ & 6 & 1,0 \\
\hline Normal diet & Enpp $1^{a s j-2 J}$ & 7 & 0,1 \\
\hline Normal diet & Enpp $1^{a s j-2 J}$ & 9 & 1,0 \\
\hline Acceleration diet & Enpp $1^{\text {asj-2J }}$ & 4 & 1,1 \\
\hline Acceleration diet & Enpp $1^{a s j-2 J}$ & 5 & 2,1 \\
\hline Acceleration diet & Enpp $1^{a s j-2 J}$ & 6 & 1,0 \\
\hline
\end{tabular}

The mice were maintained on normal or acceleration diet throughout the experiments and sacrificed at different time points as indicated for analysis of ectopic mineralization phenotypes.

Table 2: Ca, PPi/Pi ratio, TRAP and AP enzymatic activities, and CRP levels in serum/plasma of mice maintained on a normal rodent diet

\begin{tabular}{|l|l|l|l|}
\hline \multirow{2}{*}{ Parameter } & \multicolumn{4}{l|}{ Concentration/activity (mean \pm SEM) } \\
\cline { 2 - 4 } & Enpp1 $^{+/+}(\boldsymbol{n}=\boldsymbol{8})$ & Enpp1 $^{+/ \text {asj-2J }}(\boldsymbol{n}=\mathbf{1 1})$ & Enpp $^{\text {asj-2J }}(\boldsymbol{n}=\mathbf{1 0})$ \\
\hline $\mathrm{Ca}(\mathrm{mg} / \mathrm{dL})$ & $9.38 \pm 0.18$ & $9.52 \pm 0.15$ & $9.41 \pm 0.17$ \\
\hline PPi/Pi ratio $(\mathrm{x} 1,000)$ & $4.22 \pm 0.74$ & $2.96 \pm 0.38$ & $0.88 \pm 0.12^{*}$ \\
\hline TRAP activity $(\mathrm{U} / \mathrm{mL})$ & $0.05 \pm 0.01$ & $0.05 \pm 0.007$ & $0.06 \pm 0.01$ \\
\hline AP activity $(\mathrm{U} / \mathrm{L})$ & $23.63 \pm 1.77$ & $23.59 \pm 2.02$ & $42.53 \pm 1.64^{* *}$ \\
\hline $\mathrm{CRP}(\mathrm{ng} / \mu \mathrm{L})$ & $2.70 \pm 0.24$ & - & $2.37 \pm 0.25$ \\
\hline
\end{tabular}

Blood samples were collected by cardiac puncture at 12 weeks of age and Ca, Pi, CRP concentration, and TRAP and AP activities were determined in serum. PPi levels were measured in heparinized plasma. Statistical significance in comparison to Enpp $^{+/+}$mice: $* P<0.001 ; * * P<0.0001$.

Histopathological examination also indicated that the degree of tissue mineralization in asj-2J homozygous mice placed on the acceleration diet was higher than in the corresponding mice kept on control diet. Moreover, no mineralization of the cartilage, tendon, and ligament could be noted in wild type and $a s j-2 J$ heterozygous mice at 12 weeks of age (data not shown).

While ectopic mineralization was noted in asj-2J mice both on control and acceleration diet at 12 weeks of age (Set 1), we next investigated the early changes of cartilage prior to overt ectopic mineralization and the approximate time of onset of mineralization in these mice (Set 2). Mineralization of the cartilage and ligaments was first noted at around 9 weeks of age when asj-2J mice were kept on control diet, but changes of cartilage were present as early as at 6 weeks when these mice were kept on the acceleration diet. As shown in Figure 3a, Toluidine Blue $\mathrm{O}$ staining revealed large amounts of fibrocartilage on the surface of supraspinatus tendon consisting of unmineralized fibrochondrocytes. These cells mature by becoming hypertrophic towards mineralization. Following this stage of maturation, the mature fibrocartilage is invaded by ectopic immature osteophytes in joint ligaments and intervertebral discs in asj-2 J mice (Figure $3 \mathrm{~b}, 3 \mathrm{c})$. The immature osteophytes will develop into more mature osteophytes with bone when the mice age.

\section{Analysis of bone formation and resorption}

Alkaline phosphatase (AP) and tartrate-resistant acid phosphatase (TRAP) activities, reflecting bone 

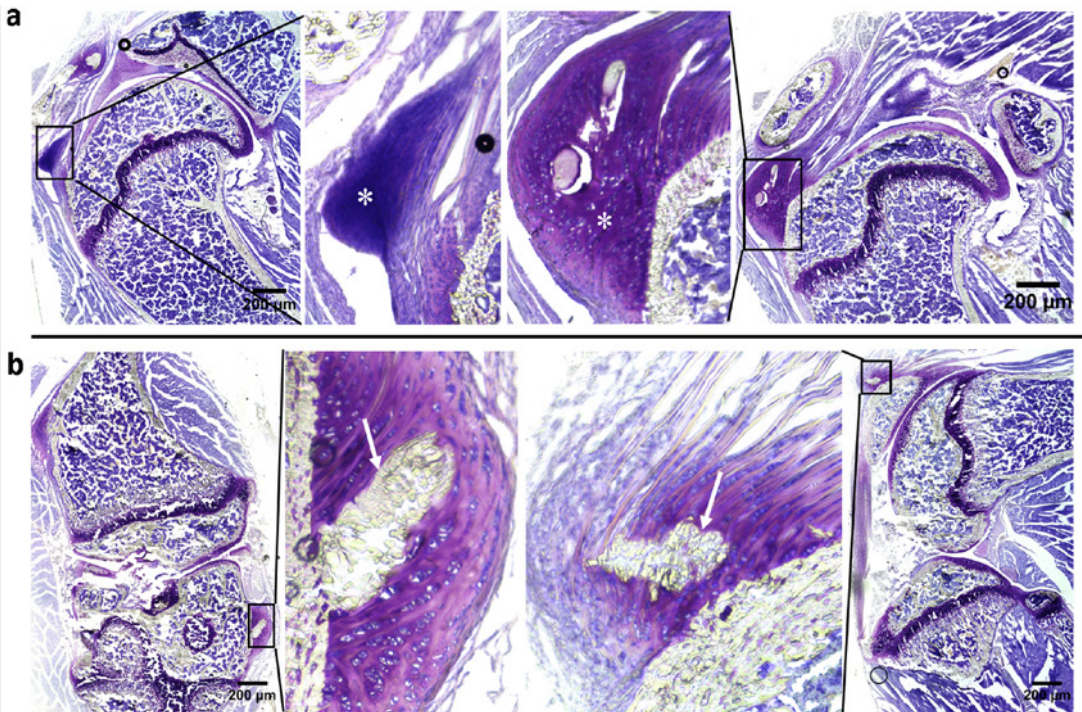
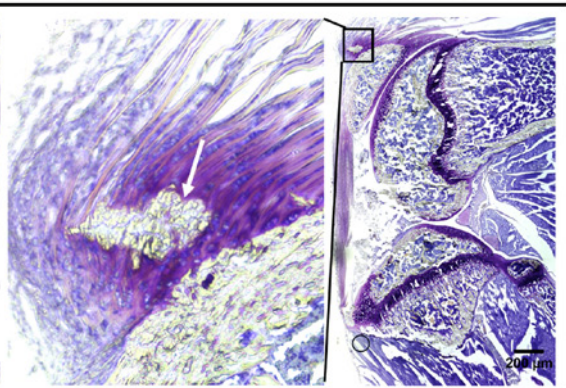

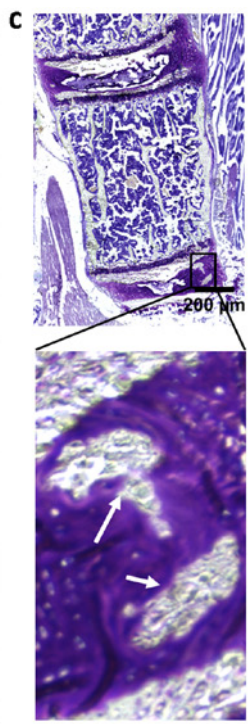

Figure 3: Aberrant fibrocartilage and presence of osteophytes preceding mineralization in asj-2 $J$ mice at 6 weeks of age (white asterisks and arrows). Toluidine Blue $O$ staining. a. Coronal section of the shoulder region shows large amount of fibrocartilage on the surface of supraspinatus tendon. $\mathbf{b}$. Coronal/sagittal section of knee shows aberrant osteophytes in joint ligament. $\mathbf{c}$. Coronal section of spine shows aberrant osteophytes in intervertebral disc.
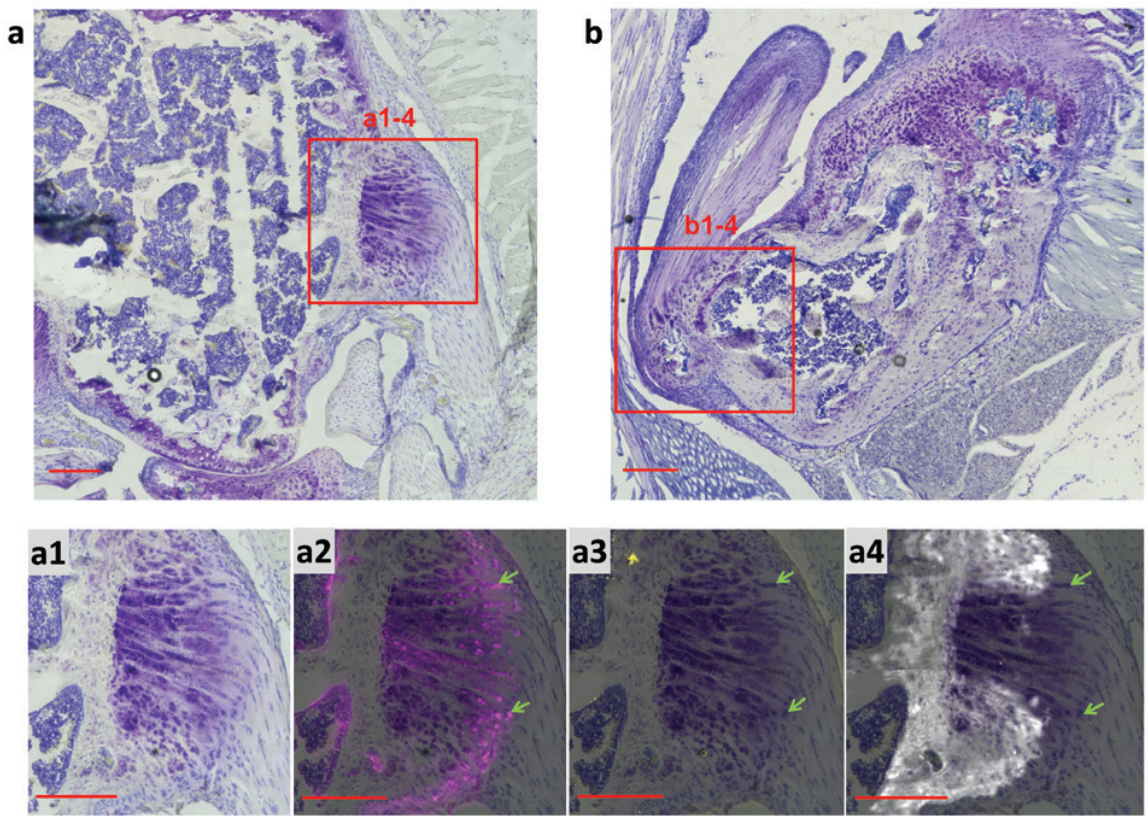

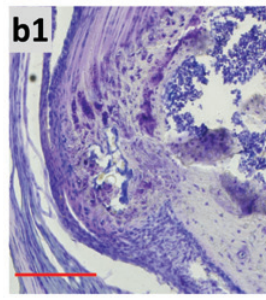

T Blue

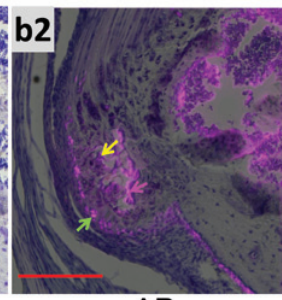

AP
T Blue

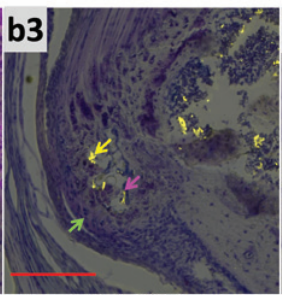

TRAP

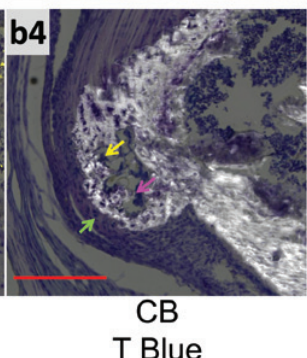

Figure 4: Osteophytes in asj-2 $J$ mice at 12 weeks of age display AP (pink) and TRAP (yellow) activities in the lateral collateral ligament in the knee (a) and the supraspinatus tendon in the shoulder (b). AP positive fibrocartilage cells (green arrows) were seen in the unmineralized regions (non-CB) of the shoulder osteophyte and in the less mature knee osteophyte. The shoulder osteophyte was more mature with TRAP positive osteoclasts (yellow arrows) and AP positive osteoblasts (pink arrows) lining the bone surfaces in the interior of the osteophyte. T Blue, Toluidine Blue O stain; AP, alkaline phosphatase; TRAP, tartrate-resistant acid phosphatase; $\mathrm{CB}$, Calcein Blue stain for mineral. Scale bar $=200 \mu \mathrm{m}$. 
formation and bone resorption [14], respectively, were analyzed at the lateral and collateral ligament in the knee and the supraspinatus tendon in the shoulder, sites of ectopic mineralization in the $a s j-2 J$ mice. AP positive osteoblasts and fibrocartilage cells were seen on frozen sections in mineralized osteophytes. Specifically, AP positive osteoblasts line the bone surfaces in the interior of the osteophyte, while AP positive fibrocartilage cells were also seen in the unmineralized regions of osteophyte. TRAP activity, a marker of osteoclasts of bone resorption, could be observed in more mature osteophytes near the supraspinatus tendon in the shoulder, but not in less mature osteophytes in the knee (Figure 4). The positive AP and TRAP stains suggested that bone formation and bone resorption were active processes at the sites of cartilage, tendon and ligament leading to ectopic mineralization.

We further examined AP and TRAP activities in serum of 12-week-old $a s j-2 J$ mice by a colorimetric method. The results indicated that serum TRAP activity was not altered; however, there was a significant increase in AP serum activity in asj-2J mice in comparison to the wild type littermates (Table 2), indicating increased bone formation rate in these mice.

\section{Serum analysis}

To examine potential inflammation in asj-2J mice, serum C-reactive protein (CRP) levels in 12-week-old $a s j-$ $2 J$ mice and their wild type counterparts were determined by an ELISA. There was no significant difference between these two groups (Table 2).

The asj-2J mice were further characterized by analyzing the calcium levels and the $\mathrm{PPi} / \mathrm{Pi}$ ratio in plasma/serum when kept on normal diet. As shown in Table 2 and reported previously [9], serum calcium levels were not altered, but $\mathrm{PPi} / \mathrm{Pi}$ ratio was significantly reduced, indicating perturbed homeostasis promoting ectopic mineralization in these mice.

\section{DISCUSSION}

\section{Connective tissue biochemistry and pathology of cartilage, tendons and ligaments}

\author{
Cartilage \\ Cartilage, flexible connective tissue providing \\ structural integrity to a number of tissues, is derived \\ from the mesoderm layer. There are three broad \\ categories of cartilage (elastic cartilage, hyaline cartilage \\ and fibrocartilage) with different extracellular matrix \\ composition and distinct tissue distribution. Elastic \\ cartilage is present in the outer ear, Eustachian tube \\ and epiglottis, the principal component being elastin.
}

Fibrocartilage is present in meniscii, intervertebral discs, and tendon/ligament entheses, and it consists of a mixture of type I and II collagens and proteoglycans. Hyaline cartilage is a highly specialized connective tissue in diarthrodial joints with the principal function being to provide a smooth surface for articulation and to facilitate movement with low frictional coefficient. Consequently, injury to articular cartilage can cause significant musculoskeletal morbidity resulting in osteoarthritis $[15,16]$. In some cases, osteoarthritis is facilitated by calcification of the cartilage, resulting in rupture and detachment of the articular cartilage as a result of trauma. Thus, cartilage mineralization can have serious consequences with little, if any, regenerative repair capacity, often necessitating joint replacement of knees or hips.

\section{Tendons}

Of the millions of musculoskeletal injuries reported in the United States per year, roughly $50 \%$ of cases involve injuries to the soft tissues, including tendons and ligaments [17]. Tendons consist of fibrous connective tissue composed primarily of densely packed collagen fiber bundles aligned parallel to the longitudinal tendon axis and surrounded by a tendon sheath. Collagen, predominantly type I collagen, constitutes $\sim 75 \%$ of the dry weight of tendon [18]. Lacerations, ruptures or inflammation to the tendon cause marked morbidity and have a major impact on work and recreational activities of the affected individuals. Tendon repair and regenerative processes are slow and often lead to extensive scarring and adhesions.

\section{Ligaments}

Skeletal ligaments are dense bands of collagenous tissue spanning joints to become anchored to the bones [19]. One of the main functions of ligaments is to mechanically stabilize joints during normal range of motion. The major forms of trauma to the ligaments result in ruptures which depending on the severity and location may or may not heal spontaneously. The healing tissues leads to scar formation and major changes in the overall composition, tissue architecture and function from the original ligament [20]. Mineralization of ligaments can also be noted in a number of clinical situations, often as a sequela to inflammation. Thus, trauma and inflammation can cause lesions to the ligaments and tendons, often resulting in dysfunction and chronic clinical sequela.

\section{Mineralization of cartilage, tendons and ligaments in asj- $2 J$ mice}

A spontaneous mutant mouse, asj-2J, was recently identified to develop extensive soft tissue mineralization, with the primary focus of the previous studies being on the skin and cardiovascular system [9]. Early observations in 
allelic series of single gene mutations in Enppl in mouse models suggested that there is mineralization also affecting joints and perispinal ligaments [21-24]. In this study, we examined the $a s j-2 J$ mice for cartilage and collagen-rich tendon and ligament mineralization first with standard histopathologic approaches. These studies demonstrated extensive mineralization of cartilages in the outer ear (elastic cartilage) and the trachea (hyaline cartilage). However, examination of fibrocartilage in the joints and mineralization in periarticular tendons and ligaments in close proximity to the bones posed technical difficulties due to our initial inability to section mineralized bone without perturbing the microarchitecture. For this reason, we resorted to the use of a novel, recently developed cryo-sectioning technique, which allows sectioning of mineralized tissues without decalcification [12, 13]. These sections retain their morphology and can be readily examined by staining with Toluidine Blue $\mathrm{O}$ and Alizarin red. The results demonstrated extensive mineralization of articular cartilages as well as adjacent ligaments in the knees and Achilles tendon in 12-week-old asj-2J mice. The mineralization process was accompanied with positive alkaline phosphatase and tartrate-resistant acid phosphatase activities on the frozen sections at the sites of ectopic mineralization. These mice also demonstrated increased alkaline phosphatase activity in their serum thus explaining the formation of mineral deposits in soft connective tissues.

A number of mouse models with ENPP1 deficiency have been previously reported [21-24], and one of them, $t$ tw (tiptoe walking mouse), has been reported to demonstrate mineralization of posterior longitudinal ligament of the spine [22]. This naturally occurring mouse is caused by a nonsense mutation p.Gly568* in the Enppl gene and is, therefore, similar to asj-2J mouse. The mineralization process in these mice is apparently elicited by reduced PPi/Pi ratio. Under normal homeostatic conditions, a certain level of PPi is required to prevent precipitation of calcium and phosphate in the form of hydroxyapatite in soft tissues [25]. The asj-2J mice are homozygous for a large deletion/insertion mutation in the Enppl gene, and as a result of absent ENPP1 activity, the plasma levels of PPi were reduced in these mice to about $21 \%$ of the control mice [9]. Thus, the $a s j-2 J$ mice serve as a genetic model of ectopic soft tissue mineralization. It should be noted that this mineralization process could be accelerated by keeping the mice on a so-called "acceleration diet", enriched in phosphate and reduced in magnesium content. Thus, the ectopic mineralization in these mice reflects interactions of several factors, including genetic background and diet, at the genome/ environment interface.

The asj-2 $J$ mouse serves as a model for GACI by demonstrating excessive vascular mineralization. Evaluation of the presence of mineralization of cartilage, ligaments and tendons in GACI patients is difficult because these patients frequently die within a few months of life due to vascular complications. However, some of these individuals survive and abnormal calcifications of ear cartilage and cervical fusions have been reported [26]. Finally, it should be noted that $a s j-2 J$ mouse has similarities with $t t w$ mouse which serves as a model of a relatively common human disease, ossification of the posterior longitudinal ligament of the spine (OPLL) [27, 28]. Thus, these mouse models serve as a platform to develop novel treatments for these, currently intractable, ectopic mineralization disorders.

\section{MATERIALS AND METHODS}

\section{Experimental procedures}

\section{Animals and diet}

Enpp $1^{a s j-2 J}$ mice (asj-2J mice) on a BALB/cJ background were obtained from The Jackson Laboratory (Bar Harbor, ME). Enpp $1^{+/+}$as well as heterozygous and homozygous asj-2J mutant mice were generated from heterozygous mattings. Mice were genotyped and maintained in a climate-controlled environment either on normal laboratory diet (Laboratory Autoclavable Rodent Diet 5010; PMI Nutritional International, St. Louis, MO) or fed an 'acceleration diet' (Rodent diet TD.00442, Harlan Teklad, Madison, WI), which we have previously shown to accelerate the ectopic mineralization in $a s j-2 J$ mice [9]; this diet is enriched in phosphorus $(2 \mathrm{x})$ and has reduced magnesium $(20 \%)$ content. The groups of mice characterized by genotype and dietary treatment are described in Table 1. All animal experiments were approved by Institutional Animal Care and Use Committee of Thomas Jefferson University. Proper handling and care were followed according to the Animal Welfare Policies of the Public Health Service of the USA.

\section{Histological analysis of soft connective tissues}

Histological analysis was performed on the outer ears and trachea of Enpp1 $1^{+/+}$, Enpp $1^{+/ a s j-2 J}$, and Enpp $1^{a s j-}$ ${ }^{2 J}$ mice. Tissues were fixed in $10 \%$ phosphate-buffered formalin, routinely processed, and embedded in paraffin. Tissues were sectioned $(7 \mu \mathrm{m})$ and stained with Alizarin red and Hematoxylin \& Eosin (H\&E) using standard procedures. After staining, the samples were examined under light microscopy for mineralization.

\section{Cryohistological analysis of bone and cartilage}

Forelimbs, hind limbs and lumbar vertebra from euthanized mice were fixed in $10 \%$ phosphate-buffered 
formalin for 2 days at $4^{\circ} \mathrm{C}$, transferred to $30 \%$ sucrose in PBS overnight at $4^{\circ} \mathrm{C}$, and then embedded in O.C.T. Compound (Tissue-Tek, Sakura, Japan). The knee was cut in the sagittal and coronal planes to capture articular cartilage and menisci. The ankle was cut in the sagittal plane, the shoulder and foot were cut in the coronal plane to investigate the articular cartilage. The lumbar vertebrae were cut in sagittal and coronal planes to investigate fibrocartilage in intervertebral discs. All sections were made from undercalcified joints using cryofilm IIC tape (Section Lab Co. Ltd, Hiroshima, Japan), which maintains morphology of mineralized sections. The taped sections were glued to microscope slides, with tissue side up, using UV adhesive glue (Norland Optical Adhesive 63, Norland Products Inc., Cranbury, NJ) and rehydrated prior to staining [12].

\section{Staining and imaging}

Because the cryofilm tape adheres to the tissue and allows for the coverslip to be removed between imaging steps without damaging the section, each section was stained up to 4 times. The order of staining included Calcein Blue, Tartrate-resistant acid phosphatase (TRAP), Alkaline Phosphatase (AP), and Toluidine Blue O staining, on the same section.

\section{Calcein Blue staining}

Slides were stained with calcein blue to label mineral deposition.

\section{TRAP staining}

Using $0.92 \%$ sodium acetate anhydrous and $1.14 \%$ sodium tartrate dibasic dehydrate make fresh TRAP reaction buffer, adjust $\mathrm{pH}$ to 4.2 . Sections were incubated in TRAP buffer for $10 \mathrm{~min}$ and then incubated with Elf97 substrate (Life Tech, Grand Island, NY) in buffer for 5 min under UV light. The Elf97 substrate generates a yellow fluorescent signal when cleaved by TRAP. The TRAP signal was imaged using Zeiss Axio Scan Z1 scanning microscope (Jena, Germany).

\section{AP staining}

After TRAP staining, the same slides were placed in PBS to remove cover slips. Sections were incubated in AP buffer (100 mM Tris, pH 9.5, $50 \mathrm{mM} \mathrm{MgCl}_{2}, 200 \mathrm{mM}$ $\mathrm{NaCl}$ ) for $10 \mathrm{~min}$ and then incubated with AP substrate, 1x Naphthol AS-MX phosphate, 1x Fast red (Sigma Aldrich, St. Louis, MO) for $5 \mathrm{~min}$ at room temperature. The AP signal was imaged using a TRITC filter (Chroma Technology Corp., Bellows Falls, VT).

\section{Toluidine Blue $O$ staining}

The same slides were stained with $0.025 \%$ Toluidine Blue $\mathrm{O}$, and re-imaged.

\section{Quantification of calcium and phosphate}

The calcium content in serum was determined colorimetrically by the $\sigma$-cresolphthalein complexone method (Calcium (CPC) Liquicolor; Stanbio Laboratory, Boerne, TX). The phosphate concentration of serum was determined with Malachite Green Phosphate Assay kit (BioAssay Systems, Hayward, CA).

\section{Inorganic pyrophosphate assay}

Whole blood was collected into heparin coated blood collection tubes to separate plasma from cells by centrifugation. The plasma was collected by filtration through a Centrisart I 300-kDa mass cutoff filter (Sartorius, New York, NY), and stored at $-80^{\circ} \mathrm{C}$ until further processing. The concentration of inorganic pyrophosphate (PPi) was determined in plasma with an enzymatic assay using uridine-diphosphoglucose (UDPG) pyrophosphorylase, with modifications, as described previously [10].

\section{TRAP and AP enzymatic activities and C-reactive protein (CRP) level}

Whole blood was collected by cardiac puncture and serum was separated from whole blood by centrifugation, $2,200 \mathrm{x}$ g at $4^{\circ} \mathrm{C}$ for $15 \mathrm{~min}$. The TRAP enzyme activity in serum was determined colorimetrically by the TRAP Assay Kit (TaKaRa, Kusatsu, Japan). The AP enzyme activity in serum was determined colorimetrically by the Alkaline Phosphatase Assay Kit (Abcam, Cambridge, MA). The serum levels of CRP were tested by Mouse C-Reactive Protein (CRP) ELISA kit (Life Diagnostics, West Chester, PA).

\section{Statistical analysis}

Statistical analyses were performed using twosided Kruskal-Wallis nonparametric test and $p<0.01$ was considered to indicate a statistically significant difference. All statistical computations were completed using SPSS (version 15.0, Chicago, IL) and GraphPad PRISM (version 5.01, San Diego, CA).

\section{Abbreviations}

GACI, generalized arterial calcification of infancy; asj, ages with stiffened joints; AP, alkaline phosphatase; TRAP, tartrate-resistant acid phosphatase; PPi, pyrophosphate; Pi, phosphate. 


\section{ACKNOWLEDGMENTS}

The authors thank Joshua Kingman for helpful advice, Yoorock Suh and Dian Wang for technical assistance. Carol Kelly assisted in manuscript preparation. The authors thank Dr. Herbert Pratt for providing mice.

\section{CONFLICTS OF INTEREST}

There are no Conflict of Interests to disclose.

\section{GRANT SUPPORT}

This study was supported by NIH grants R01AR55225 (JU and JPS), K99AR067283 (NAD), and K01AR064766 (QL).

\section{REFERENCES}

1. Budoff MJ, Shaw LJ, Liu ST, Weinstein SR, Mosler TP, Tseng PH, Flores FR, Callister TQ, Raggi P and Berman DS. Long-term prognosis associated with coronary calcification: observations from a registry of 25,253 patients. J Am Coll Cardiol. 2007; 49:1860-1870.

2. Chander S and Gordon P. Soft tissue and subcutaneous calcification in connective tissue diseases. Curr Opin Rheumatol. 2012; 24:158-164.

3. Giachelli CM. Ectopic calcification: new concepts in cellular regulation. Z Kardiol. 2001; 90 Suppl 3:31-37.

4. Sprecher E. Familial tumoral calcinosis: from characterization of a rare phenotype to the pathogenesis of ectopic calcification. J Invest Dermatol. 2010; 130:652-660.

5. Li Q and Uitto J. Mineralization/anti-mineralization networks in the skin and vascular connective tissues. Am J Pathol. 2013; 183:10-18.

6. Uitto J, Li Q and Jiang Q. Pseudoxanthoma elasticum: molecular genetics and putative pathomechanisms. J Invest Dermatol. 2010; 130:661-670.

7. Nitschke $Y$ and Rutsch F. Generalized arterial calcification of infancy and pseudoxanthoma elasticum: two sides of the same coin. Front Genet. 2012; 3:302.

8. Rutsch F, Ruf N, Vaingankar S, Toliat MR, Suk A, Hohne W, Schauer G, Lehmann M, Roscioli T, Schnabel D, Epplen JT, Knisely A, Superti-Furga A, McGill J, Filippone M, Sinaiko AR, et al. Mutations in ENPP1 are associated with 'idiopathic' infantile arterial calcification. Nature Genet. 2003; 34:379-381.

9. Li Q, Pratt CH, Dionne LA, Fairfield H, Karst SY, Sundberg JP and Uitto J. Spontaneous asj-2J mutant mouse as a model for generalized arterial calcification of infancy: A large deletion/insertion mutation in the Enpp1 gene. PLoS One. 2014; 9:e113542.

10. Li Q, Guo H, Chou DW, Berndt A, Sundberg JP and Uitto J. Mutant Enpplasj mouse as a model for generalized arterial calcification of infancy. Dis Model Mech. 2013; 6:12271235 .

11. Dijkgraaf LC, de Bont LG, Boering $G$ and Liem RS. Normal cartilage structure, biochemistry, and metabolism: a review of the literature. J Oral Maxillofac Surg. 1995; 53:924-929.

12. Dyment NA, Hagiwara Y, Jiang X, Huang J, Adams DJ and Rowe DW. Response of knee fibrocartilage to joint destabilization. Osteoarthritis Cartilage. 2015; 23:996-1006.

13. Dyment NA, Breidenbach AP, Schwartz AG, Russell RP, Aschbacher-Smith L, Liu H, Hagiwara Y, Jiang R, Thomopoulos S, Butler DL and Rowe DW. Gdf5 progenitors give rise to fibrocartilage cells that mineralize via hedgehog signaling to form the zonal enthesis. Dev Biol. 2015; 405:96-107.

14. Cepelak I and Cvoriscec D. Biochemical markers of bone remodeling - review. Biochemia Medica. 2009; 19:17-35.

15. Sophia Fox AJ, Bedi A and Rodeo SA. The basic science of articular cartilage: structure, composition, and function. Sports Health. 2009; 1:461-468.

16. Kouri Sr. JB. Cartilage mineralization and cell death within experimental osteoarthritis. Osteoarthr Cartilage. 2014; 22:S362.

17. James R, Kesturu G, Balian G and Chhabra AB. Tendon: biology, biomechanics, repair, growth factors, and evolving treatment options. J Hand Surg Am. 2008; 33:102-112.

18. Tkocz C and Kuhn K. The formation of triple-helical collagen molecules from alpha-1 or alpha-2 polypeptide chains. Eur J Biochem. 1969; 7:454-462.

19. Frank CB. Ligament structure, physiology and function. J Musculoskelet Neuronal Interact. 2004; 4:199-201.

20. Shrive N, Chimich D, Marchuk L, Wilson J, Brant R and Frank C. Soft-tissue "flaws" are associated with the material properties of the healing rabbit medial collateral ligament. J Orthop Res. 1995; 13:923-929.

21. Mackenzie NC, Huesa C, Rutsch F and MacRae VE. New insights into NPP1 function: lessons from clinical and animal studies. Bone. 2012; 51:961-968.

22. Okawa A, Nakamura I, Goto S, Moriya H, Nakamura Y and Ikegawa S. Mutation in Npps in a mouse model of ossification of the posterior longitudinal ligament of the spine. Nature Genet. 1998; 19:271-273.

23. Sali A, Favaloro JM, Terkeltaub R and Goding JW. (1999). Germline deletion of the nucleoside triphosphate pyrophosphohydrolase (NTPPPH) plasma cell membrane glycoprotein-1 (PC-1) produces abnormal calcification of periarticular tissues. In: Vanduffel L LR, ed. Ecto-ATPases and related ectoenzymes. (Maastricht, The Netherlands: Shaker Publishing), pp. 267-282.

24. Babij P, Roudier M, Graves T, Han CY, Chhoa M, Li CM, Juan T, Morony S, Grisanti M, Li X, Yu L, Dwyer D, Lloyd DJ, Bass MB, Richards WG, Ebeling C, et al. New variants in the Enpp1 and Ptpn6 genes cause low BMD, crystalrelated arthropathy, and vascular calcification. J Bone Min 
Res. 2009; 24:1552-1564.

25. Ruf N, Uhlenberg B, Terkeltaub R, Nurnberg P and Rutsch F. The mutational spectrum of ENPP1 as arising after the analysis of 23 unrelated patients with generalized arterial calcification of infancy (GACI). Hum Mutat. 2005; 25:98.

26. Nitschke Y, Baujat G, Botschen U, Wittkampf T, du Moulin M, Stella J, Le Merrer M, Guest G, Lambot K, TazarourtePinturier MF, Chassaing N, Roche O, Feenstra I, Loechner K, Deshpande C, Garber SJ, et al. Generalized arterial calcification of infancy and pseudoxanthoma elasticum can be caused by mutations in either ENPP1 or ABCC6. Am J Hum Genet. 2012; 90:25-39.

27. Ono K, Ota H, Tada K, Hamada H and Takaoka K. Ossified posterior longitudinal ligament. A clincopathologic study. Clin Orthop. 1977; 184:71-84.

28. Tsuyama N. Ossification of the posterior longitudinal ligament of the spine. Clin Orthop Relat Res. 1984; 184:7184. 